\title{
The vote Package: Single Transferable Vote and Other Electoral Systems in $\mathbf{R}$
}

\author{
by Adrian E. Raftery, Hana Ševčíková and Bernard W. Silverman
}

\begin{abstract}
We describe the vote package in R, which implements the plurality (or first-past-the-post), two-round runoff, score, approval, and Single Transferable Vote (STV) electoral systems, as well as methods for selecting the Condorcet winner and loser. We emphasize the STV system, which we have found to work well in practice for multi-winner elections with small electorates, such as committee and council elections, and the selection of multiple job candidates. For single-winner elections, STV is also called Instant Runoff Voting (IRV), Ranked Choice Voting (RCV), or the alternative vote (AV) system. The package also implements the STV system with equal preferences, for the first time in a software package, to our knowledge. It also implements a new variant of STV, in which a minimum number of candidates from a specified group are required to be elected. We illustrate the package with several real examples.
\end{abstract}

\section{Introduction}

The vote package implements several electoral methods: plurality voting, approval voting, score voting, Condorcet methods, and Single Transferable Vote (STV) methods (Ševčíková et al., 2021).

In developing the package, we were motivated particularly by the needs of organizations with small electorates, such as learned societies, clubs, and university departments, who may need to elect more than one person in a given election. In the early 1980s, one of us (BWS) was a member of the Royal Statistical Society (RSS) Council. At that time, six members of the Council were elected at a time. A nominating committee nominated six candidates, and the RSS membership as a whole voted, with each member allowed to vote for up to six candidates, and the six candidates with the most votes being elected. Usually, there were only the six nominated candidates, but that year a seventh candidate stood on a platform different from that of the "official" candidates. This candidate received votes from about a quarter of the electorate but was not elected because the other three-quarters of the members voted as a block for the six candidates proposed by the nominating committee.

This was viewed as unsatisfactory because the seventh candidate's position was not represented on the Council, even though it had substantial support among the RSS membership. This led the RSS Council to undertake a study of electoral methods for multi-winner elections, with a view to adopting a more representative system. They selected the Single Transferable Vote (STV) method, which was then adopted for Council elections, initially using a program in the Pascal programming language developed by Hill et al. (1987). In the next election, held under STV, the seventh candidate stood again and was elected. STV has been used since then to elect the RSS Council.

In 2002, the Institute of Mathematical Statistics (IMS), the leading international association of academic mathematical statisticians, considered the same issue and came to the same conclusion, also adopting STV for its Council elections. They used an R program developed by BWS (Silverman, 2002, 2003), who was also then the IMS President. This R program became the core of the vote package that we are describing here. This STV electoral method has been used since then by the IMS.

Since then, another one of us (AER) has implemented the STV method in the context of small electorates selecting or ranking multiple candidates, such as nominating committees selecting multiple awardees for a prize, or academic departments selecting job candidates for interviews. Those involved have generally reported finding the method satisfactory. This experience has led to several modifications of the program that are also implemented in the package.

Our implementation and discussion of STV and other systems is aimed particularly at those involved in non-party-political elections and decisions, such as those outlined above. Questions of what approaches are or are not desirable for national elections are matters of political science beyond the scope of this paper, which is not intended to advocate for or against the use of any particular voting systems in that context. However, a brief review may be informative.

The USA and the UK, for their national legislatures, almost entirely use the plurality, "first past the post" or "winner takes all" system, where the leading candidate in each district is elected. The Electoral College for the US presidency is also elected this way, but with an election between slates rather than individuals, in all states except Maine and Nebraska. On the other hand, the majority of countries use some system that (in principle at least) aims for the elected body to represent the views of the wider electorate proportionately, either over the country as a whole or within larger electoral districts. However, pure proportional systems are fairly unusual, for example because in nationwide 
proportional systems there is often a threshold below which a party will not have any representation. The Single Transferable Vote system is used to elect the parliaments or national assemblies of the Republic of Ireland, Northern Ireland, and Malta, as well as upper houses and/or local assemblies in some other countries (Wikipedia, 2020c), and we draw an example from a Dublin election in the paper.

As we have said, it is not our purpose to advocate any one electoral method, and indeed it is well known that there is no one method that dominates all others given a reasonable set of criteria, according to the impossibility theorems of Arrow (1963), Gibbard (1973) and Satterthwaite (1975). Indeed, method choice can depend on the purpose of the election, and a method that works well for one purpose (such as representing the views of the electorate), may not be best for others (such as electing an effective team to work together) (Syddique, 1988). As a result, we have implemented multiple electoral methods in the package. Pros and cons of a wide range of different electoral systems are described in Ace Project (2020), but these focus on nationwide political elections, whereas here we also pay attention to smaller, often non-political elections, such as those for councils and committees.

The paper is organized as follows. In Section 2.2, we describe the plurality, two-round runoff, approval, score, and Condorcet vote-counting methods. In Section 2.3, we describe the STV method, including the first software implementation of the equal preference STV method, to our knowledge. This also describes a new variant of STV, which enforces minimal representation of a marked group. In Section 2.4, we describe three multi-winner elections with electorates of different sizes: an election from one constituency in the 2002 Irish General Election, an election of the IMS Council, and a vote to select job candidates by a university department. We conclude in Section 2.5 with the discussion of issues including other $R$ packages for vote-counting.

\section{Electoral methods}

In this section, we describe several electoral methods and how they are implemented in the vote package. We defer description of STV to Section 2.3.

We first illustrate the results here with the toy food_election dataset:

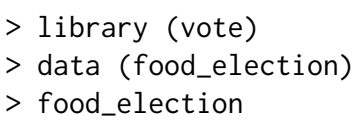

In this toy dataset, voters were asked to rank the options in order of preference. They gave only their first two preferences, although they could have given more; an NA indicates that no preference was expressed.

\section{Plurality voting}

Plurality voting, or First-Past-The-Post, is used for single-winner elections, such as elections to the House of Representatives in the USA or the House of Commons in the UK. Each voter votes for one candidate, and the candidate with the most votes wins. 

count:

To implement this with our toy dataset, we first converted it to a dataset where only first preferences

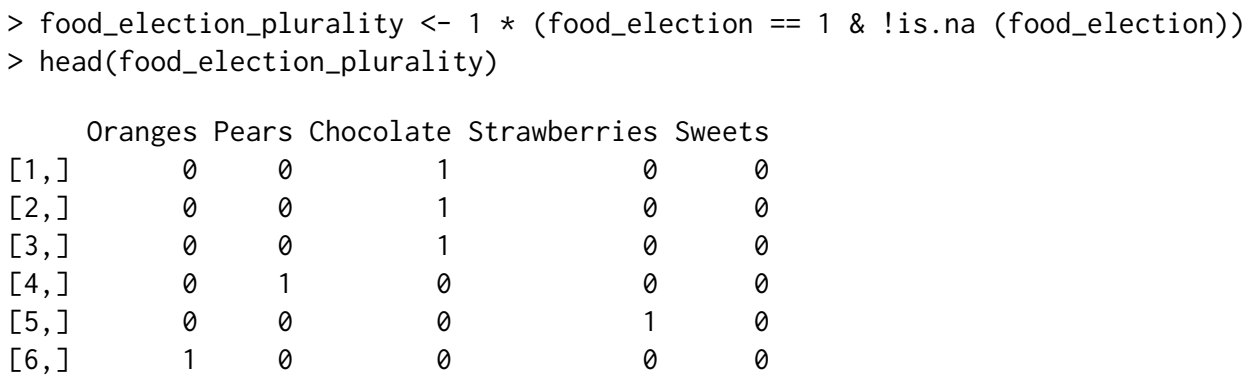

We then counted the votes using the plurality command:

$>$ plurality (food_election_plurality)

Results of Plurality voting

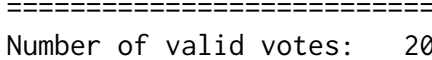

Number of invalid votes: 0

Number of candidates: 5

Number of seats:

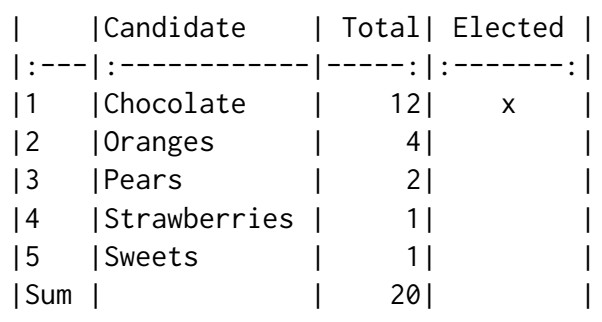

Elected: Chocolate

Plurality voting has the advantage of simplicity. In political elections, it tends not to yield results that are in direct proportion to support among the voters but to amplify pluralities when compared to proportional voting systems, which merge single-winner districts into larger multi-member groups. In general, any large party which has strong support across a large number of electoral districts will do well under plurality voting, while smaller parties or interests will tend to be underrepresented numerically, especially if they are evenly or thinly spread. This may mean that important interests are not represented, while on the other hand, it may present a barrier to the traction of extremist groups. The US Electoral College is, in nearly all states, elected by a plurality voting system, with multiple members, all being elected simultaneously.

Plurality voting in individual-member districts tends to lead to one-party governments with working majorities, even when the leading party does not achieve half of the popular vote. It also allows districts to be smaller to facilitate direct contact between a voter and their representative and identifies each representative more closely with all the voters in their district.

Another effect of plurality voting can be to "waste" the votes of those who live in highly polarised districts, because they win their particular district by a very wide margin; this seems to be a deliberate feature of much redistricting in the USA. In non-political elections in the smaller contexts of primary concern in this paper, there is little or no need for a stable one-"party" result, and the desirability of closer proportional representation of the views of the voters is less contentious, and so there is likely to be a clearer case for using other voting systems wherever possible.

\section{Two-round runoff voting}

Two-round systems are also used for single-winner elections. In the first round, voters vote for their first preference. If no candidate gets a majority, there is a second round that involves the top two candidates. Voters vote again, and the candidate getting more votes wins.

In the vote package, we implemented a variant of this system that can be counted in a single pass over the data. Each voter ranks the candidates in order of preference. The first round takes place as 
described. The second round is counted as if voters voted for the remaining candidate for which they had a higher preference.

To illustrate the two-round runoff system, we modify the food election data by removing voters $12-15$, so that Chocolate does not have a majority on the first round:

$>$ food_election3 <- food_election[-c(12:15), ]

$>$ tworound.runoff (food_election3)

Results of two-round-runoff voting

$=======================$
Number of valid votes: 16

Number of invalid votes: 0

Number of candidates: 5

Number of seats:

\begin{tabular}{|c|c|c|c|c|c|c|}
\hline $\begin{array}{l}1 \\
1:-\end{array}$ & $\begin{array}{l}\text { | Candidate } \\
-\mid:-\end{array}$ & $\mid$ Total $\mid$ & ercent| & & $\begin{array}{l}\text { ercent } \mid \\
-----\mid\end{array}$ & \\
\hline 11 & |Oranges & $4 \mid$ & 25.01 & 61 & 42.91 & \\
\hline 12 & |Pears & $2 \mid$ & $12.5 \mid$ & 01 & 0.01 & \\
\hline 13 & Chocolate & 81 & 50.01 & 81 & 57.11 & $\mathrm{x}$ \\
\hline 14 & Strawberries & $1 \mid$ & 6.21 & 01 & 0.01 & \\
\hline 15 & | Sweets & 11 & 6.21 & 01 & 0.01 & \\
\hline Sum & I & $16 \mid$ & 100.01 & $14 \mid$ & 100.01 & \\
\hline
\end{tabular}

Elected: Chocolate

We see that no candidate got a majority on the first round, although Chocolate came close. In the second round, the two top vote-getters, Chocolate and Oranges, squared off, and Chocolate won.

In the tworound.runoff function, a tie in either the first or the runoff round is resolved by random draw. A random seed can be specified so that the results are replicable.

Two-round elections are quite common, most famously for French presidential elections since 1965. In practice, it is usually carried out by voters actually voting twice, rather than ranking candidates as here. An exception to it is a special case of the two-round runoff, called supplementary voting, where voters give only their first and second preferences on one ballot, the same way as happened in our food example. Supplementary voting is used, for example, in electing mayors in England, including the Mayor of London (London Elects, 2020).

The two-round runoff system differs from plurality voting in that voters for candidates with low levels of support can change their votes to one of the leading candidates so that they can express support for a possibly less popular first choice without their vote being "wasted". Of course, the choice between the two finalists shares some of the aspects of plurality voting.

\section{Approval voting}

Approval voting was advocated by Brams and Fishburn (1978). In this system, voters vote for as many candidates as they wish. It has been most often advocated for single-winner elections, in which case the winner is the candidate with the most votes (Brams and Fishburn, 2007). A direct extension to multi-winner elections with $m$ winners is that voters vote in the same way, and the $m$ candidates with the most votes win.

Counting the votes is simple. The argument nseats determines the number of winners $m$ :

$>$ food_election_approval <- 1 * ! is.na (food_election)

$>$ approval (food_election_approval, nseats $=2$ )

Results of Approval voting

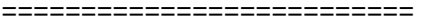

Number of valid votes: 20

Number of invalid votes: 0

Number of candidates: 5

Number of seats: 2

| |Candidate | Total| Elected |

$|:---|:------------|-----:|:-------: \mid$ 


\begin{tabular}{|c|c|c|c|c|}
\hline $\mid 1$ & |Chocolate & I & 121 & $\mathrm{x}$ \\
\hline 12 & Strawberries & I & 91 & $\mathrm{x}$ \\
\hline 13 & |Oranges & i & 61 & \\
\hline 14 & | Sweets & 1 & 51 & \\
\hline 15 & |Pears & | & 21 & \\
\hline Sum & 1 & | & $34 \mid$ & \\
\hline
\end{tabular}

Elected: Chocolate, Strawberries

Approval voting for multi-winner elections has been criticized on various grounds, e.g., Hill (1988), and indeed in the book by Brams and Fishburn (1983) that advocated and popularized approval voting for single-winner elections. For elections in which there are parties or slates of candidates, it would tend to lead to the election of all the members of the most supported party or slate, as happened in the RSS Council election that first motivated this work. However, one of us [AER] has participated in multi-winner elections using approval voting and has observed it to work well, particularly when there are many candidates about whom information is limited and there are no parties or slates. One example could be the early stages of job candidate selection when a long list is being whittled down to a small set of finalists.

\section{Score voting}

In the score or range voting, each voter gives each candidate a score within a prespecified range. If the voter does not give a score to a particular candidate, a corresponding prespecified score is assigned. The candidates with the lowest scores win (or the highest scores if higher scores are better). In the score function, the argument larger . wins specifies whether lower scores are better or higher scores are better. The argument max. score sets the prespecified non-vote score. Here, we illustrate score voting by applying it to the food election example, where the score is equal to the preference, a non-vote is assigned a value of 6 , and lower scores are better:

$>$ score (food_election, larger.wins $=$ FALSE, nseats $=2$, max. score $=6$ )

Results of Score voting

$====================$
Number of valid votes: 20

Number of invalid votes: 0

Number of candidates: 5

Number of seats: $\quad 2$

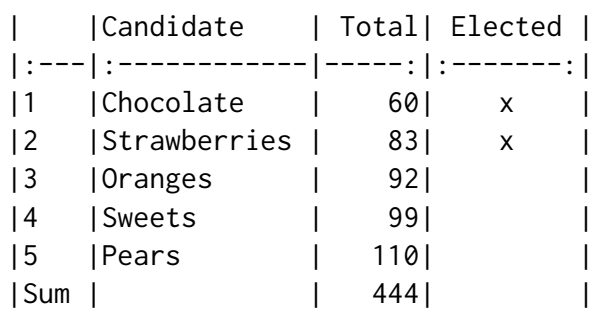

Elected: Chocolate, Strawberries

Score voting is often used by committees for purposes such as selecting grant applications to be funded. In such cases, there are often many candidates or applications to be assessed, and it would not be feasible for the voters to produce a complete ranking. Score voting is similar to multi-winner approval voting in this sense but allows for a more refined assessment by the voters. Multi-winner approval voting is actually a special case of score voting.

\section{Condorcet method}

The Condorcet method is attributed to Marquis de Condorcet (de Condorcet, 1785). It is a single-winner method where voters rank the candidates according to their preferences. The so-called Condorcet winner is the candidate who wins the majority of votes in all head-to-head comparisons. In other words, each candidate is compared pairwise to all other candidates. To become the Condorcet winner, one has to win all such comparisons. Analogously, a Condorcet loser is the candidate who loses in every pairwise comparison. 
The condorcet function can be applied directly to the food election data:

$>$ condorcet(food_election)

Results of Condorcet voting

$=======================$
Number of valid votes: 20

Number of invalid votes: 0

Number of candidates: 5

Number of seats:

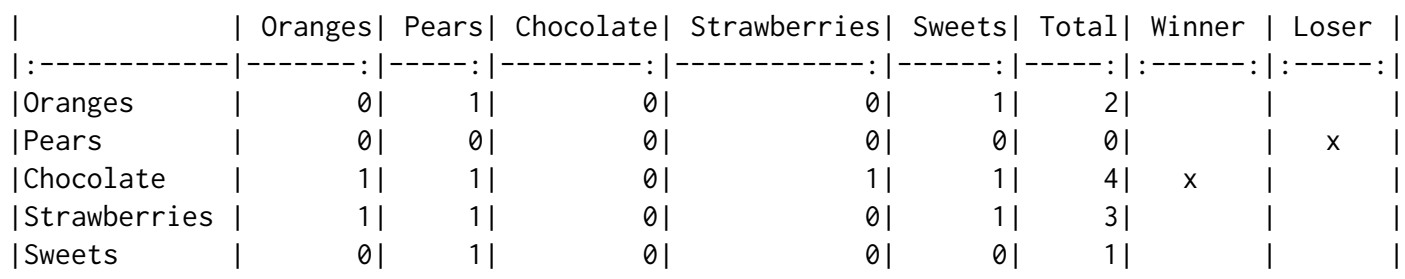

Condorcet winner: Chocolate

Condorcet loser: Pears

The output above shows the results of all the pairwise comparisons. Chocolate beat all other candidates and was, therefore, the Condorcet winner. Similarly, Pears lost against all other candidates and was thus the Condorcet loser.

The Condorcet method does not guarantee that a Condorcet winner exists. There are many different ways to deal with such a situation; see, for example, Wikipedia (2020a). Our implementation offers the possibility of a runoff (argument runoff). In this case, two or more candidates with the most pairwise wins are selected, and the Condorcet method is applied to such subset. If more than two candidates are in such a runoff, the selection is performed repeatedly until either a winner is selected or no more selection is possible.

To our knowledge, the Condorcet method is not used for governmental elections anywhere in the world. Wikipedia (2020a) cites a few private organizations that use the method, e.g., the Student Society of the University of British Columbia.

\section{Single Transferable Vote (STV)}

The Single Transferable Vote (STV) system is also referred to as Ranked Choice Voting (RCV), Instant Runoff Voting (IRV), or the Alternative Vote (AV) system for single-winner elections, and as Multi-Winner Ranked Choice Voting for multi-winner elections. One of the properties of the Single Transferable Vote system is that if any subset of candidates gets a sufficient share of the votes, anything strictly exceeding $1 /(m+1)$, where $m$ is the number of candidates to be elected, then one of this group is bound to be elected. To be precise, what is required is that a proportion above $1 /(m+1)$ of the voters have to put all the candidates in the subset at the top of their list of preferences, but it does not matter in what order. This would apply equally if the subset was a particular slate/party, or specified by some other group characteristic such as sex, race, geographical location, career stage, or subject area, even if the subset was not consciously constituted. In particular, if a candidate's proportion of the first preference votes is above $1 /(m+1)$, then that candidate will be successful.

There is also the fact that a group is not disadvantaged if more of its members stand for election, at least if their voters vote along group lines the full way down the preferences. Unlike in some other systems, they cannot cancel each other out.

When STV was adopted for the elections of the Council of the RSS in the mid-1980s and the IMS in 2002, it was hoped that it would lead to more diverse Councils than the results of the previous methods, and also that individual members, other than those chosen by the nominating committee, would feel able to stand with a real chance of being elected.

\section{STV method}

There are many descriptions of the STV system (Newland et al., 1997; Fair Vote, 2020) and its history (Hill, 1988; Tideman, 1995). The basic principle is that voters rank the candidates in order of preference. In order to be elected, a candidate must achieve the quota of $N /(m+1)+\varepsilon$, where $N$ is the total 
number of votes cast, $m$ is the number of candidates to be elected (or seats), and $\varepsilon$ is a pre-specified small positive number, often taken to be 1 when the electorate is large and 0.001 when it is small. Excess votes over the quota are appropriately downweighted and allocated to the next preference of voters. If no candidate reaches the quota, the candidate with the smallest number of votes is eliminated and his or her votes are transferred to the next preferences.

Voters are asked to rank the candidates $1,2,3, \ldots$ until they have no further preference between candidates. Thus 1 is a voter's first preference, 2 is their next choice, and so on. There is no disadvantage to higher candidates in expressing a full list of preferences; later preferences are used only when the fate of candidates given higher preferences has been decided one way or the other.

By default, a vote is considered spoiled if the preferences are not numbered consecutively, starting at 1 . However, if this is not desired, the votes can be preprocessed to be consecutive using the correct. ranking function in the vote package. A useful application of this correction is the case when a candidate has to be removed, perhaps because of having withdrawn his or her candidacy. In this case, the function remove. candidate can be used, which removes the given candidate(s) from the set of votes, and also adjusts the preferences to be consecutive. The package optionally allows the user to accept a partially correct ranking. That is, only preferences equal to or higher than the non-consecutive rankings are removed. For example, with this option, a valid version of a vote 1, 2, 3, 4, 4, 5 would be $1,2,3,0,0,0$.

Also by default, apart from the candidates not numbered at all, no ties are allowed among the numbered preferences. However, equal preferences can be allowed by using the setting equal . ranking=TRUE in the stv function, as described in more detail in Section 2.3.4.

The fact that some voters may not express a full list of preferences can be allowed for by reducing the quota in later counts ${ }^{1}$. In the vote package, the default is that the quota is reduced in later counts. However, in some STV systems (such as the electoral system in the Republic of Ireland), the quota remains constant over counts at the value that is initially defined. This is specified in the vote package by setting the argument constant. quota=TRUE in the stv function. In this implementation of STV, the last candidate is often elected without reaching the quota, which does not happen when the quota is reduced appropriately at each count.

In the vote package, the votes should be entered into a matrix or data frame, with the header containing the names of the candidates and each row the votes cast, with blank preferences being replaced by zeroes or NAs. This will often be done by entering the votes into a spreadsheet first and then reading the spreadsheet into $R$. If the data are stored in a text file, the package allows one to pass the name of the file directly into the stv function while setting the column separator in the fsep argument.

At the end of the process, the program yields a list of the successful candidates in the order in which they were elected. It also usually yields a complete ordering of the candidates. This may be useful, for example, if the purpose of the election is to select job candidates, and one wishes to have an ordered list of the initially unsuccessful candidates in case any of those selected decline the offer. Also, in some systems, candidates can claim expenses if a certain rank is achieved, which could be another motivation for a runner-up list being available.

Until the 1980s, STV elections were counted manually by physically transferring a sample of the ballot papers from the pile of the candidate being elected or eliminated, to those of the benefitting candidates. This remains the case in several long-established STV election systems, such as elections for the Dáil (the lower house of the Irish parliament). Meek $(1969,1970)$ described the form a computerbased STV system could take, and this was implemented in Pascal by Hill et al. (1987). This code was used for the RSS Council elections. A modified version was implemented in R by Silverman (2002, 2003), and this was the starting point for the current STV implementation in the vote package.

Here is the result of the food election with two candidates to be elected, using the stv function:

$>$ stv (food_election, nseats $=2$ )

Results of Single transferable vote

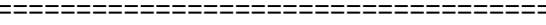

Number of valid votes: 20

Number of invalid votes: 0

Number of candidates: 5

Number of seats: 2

\footnotetext{
${ }^{1}$ In STV, the process of distributing the surplus or votes of a candidate who is elected or eliminated is referred to variously as a count, a stage, or a round. Here, we use the term count. The tabulation of the first preference votes is then called the first count.
} 


\begin{tabular}{|c|c|c|c|c|c|c|c|}
\hline | & $1 \mid$ & $2-\operatorname{trans} \mid$ & 21 & ans $\mid$ & 31 & 4-trans & $4 \mid$ \\
\hline & & & $---: \mid$ & $-: 1-$ & $----: \mid$ & $-: 1$ & $----: \mid$ \\
\hline |Quota & $6.668 \mid$ & | & $6.667 \mid$ & | & $6.667 \mid$ & | & $5.278 \mid$ \\
\hline |Oranges & $4.000 \mid$ & 0.0001 & 4.0001 & 21 & 6.0001 & 0.0001 & 6.0001 \\
\hline |Pears & $2.000 \mid$ & 0.0001 & 2.0001 & $-2 \mid$ & 1 & | & \\
\hline |Chocolate & $12.000 \mid$ & $-5.332 \mid$ & | & i & | & $\mid$ & \\
\hline Strawberries & 1.0001 & $3.555 \mid$ & $4.555 \mid$ & 01 & $4.555 \mid$ & 0.0001 & $4.555 \mid$ \\
\hline | Sweets & 1.0001 & $1.777 \mid$ & $2.777 \mid$ & 01 & $2.777 \mid$ & $-2.777 \mid$ & \\
\hline |Elected & | Chocolate| & | & | & | & | & & Oranges| \\
\hline Eliminated & i & $\mid$ & Pears| & i & Sweets| & | & \\
\hline
\end{tabular}

Elected: Chocolate, Oranges

Oranges was elected second, whereas, under the approval vote system with first and second preferences treated equally, Strawberries was elected second. This reflects the fact that Oranges had 4 first preferences, whereas Strawberries had only 1 . Under STV, a vote is credited entirely to the first preference candidate unless that candidate is elected or eliminated, in which case the second preferences come into play. Strawberries had 8 second-preference votes, all of which were from voters who voted for Chocolate first. The quota was only $56 \%$ of the votes for Chocolate, and so $44 \%$ of Chocolate's votes were transferred when Chocolate was elected. Strawberries gained 3.555 votes this way from its second preference votes, but this was not quite enough to overcome Orange's advantage in first preferences. The complete ordering of candidates can be read off the results: Chocolate, Oranges, Strawberries, Sweets, Pears. Setting the argument complete. ranking to TRUE will include the complete ordering as part of the output.

The package has several functions for visualizing the STV results, and we will illustrate these in the Examples section below. In addition, summary functions are available for the resulting objects of all voting methods in the package. In the case of stv, the summary function returns a data frame containing the table shown in the above output, which can be used for further processing, for example, for storing in a spreadsheet.

\section{Computational methods}

The algorithm used for counting STV elections using the stv function in the vote package is shown in Algorithm 1. There are only two changes needed to implement STV with equal preferences; these are shown in Section 2.3.4.

\section{Tie-breaking}

Suppose that on a given count, no candidate is elected, and a candidate needs to be selected for elimination, and that two or more candidates are tied with the smallest number of votes. Then a method is needed for choosing the one to be eliminated. The same issue arises when two candidates can be elected on the same count with the same number of votes, namely which surplus to transfer first.

Several different methods have been proposed. The Electoral Reform Society, one of the leading organizations advocating the use of STV, recommends using the Forwards Tie-Breaking Method (Newland et al., 1997, Section 5.2.5). Other methods such as Backwards Tie-Breaking, Borda TieBreaking, Coombs Tie-Breaking, or a combination of those have been proposed; see, e.g., O'Neill (2004); Kitchener (2005); Lundell (2006).

By default, the vote package uses the Forwards Tie-Breaking Method. This consists of eliminating/electing the candidate who had the fewest/most votes on the first count or on the earliest count where they had unequal votes. If the argument ties in the stv function is set to "b", the Backwards Tie-Breaking Method is used. In this case, it eliminates/elects the candidate who has the fewest/most votes on the latest count where the tied candidates had unequal votes.

There is no guarantee that a tie will be broken by either the Forwards or Backwards Tie-Breaking Method. Also, if one of these two methods does not break the tie, the other will not either because the tied candidates will have the same number of votes in all the counts so far. In particular, this will be the case whenever a tie has to be broken on the first count, and it is also relatively likely when a tie arises on the second count.

When there is a tie that Forwards and Backwards Tie-Breaking fail to break, the stv function uses a method that compares the candidates on the basis of the numbers of individual preferences. We call this the Ordered method as it creates an ordering of the candidates before the STV count begins. First, candidates are ordered by the number of first preferences. Any ties are resolved by proceeding to the 
1: procedure $\operatorname{STV}(X, m, \varepsilon)$

$$
\begin{aligned}
& \triangleright X \text { are votes of size } N \text { (number of votes) } \times M \text { (number of candidates) } \\
& D \leftarrow\{1,2, \ldots, M\} \\
& E \leftarrow\{\} \\
& F \leftarrow\{\} \\
& L \leftarrow m \\
& Y \leftarrow X \\
& c \leftarrow 0 \\
& w_{i} \leftarrow 1 \quad \forall i=1, \ldots, N \\
& \text { while } L>0 \text { do } \\
& c \leftarrow c+1 \\
& u_{i, j} \leftarrow w_{i} \delta_{Y_{i, j}}(1) \quad \forall i=1, \ldots, N, j=1, \ldots, M \quad \triangleright \text { Weighted first preferences } \\
& v_{c, j} \leftarrow \sum_{i=1}^{N} u_{i, j} \quad \forall j=1, \ldots, M \quad \triangleright \text { Sum of weighted first preferences } \\
& Q \leftarrow \sum_{j=1}^{M} v_{c, j} /(L+1)+\varepsilon \\
& \text { if } \max _{j \in D} v_{c, j} \geq Q \text { then } \\
& k \leftarrow \arg \max _{j \in D} v_{c, j} \\
& \text { if }\|k\|>1 \text { then } \\
& k \leftarrow \text { resolve.tie.for.election }(k, X, v) \\
& \text { end if } \\
& S \leftarrow\left(\max _{j \in D} v_{c, j}-Q\right) / \max _{j \in D} v_{c, j} \\
& w_{r} \leftarrow u_{r k} * S \quad \forall r \text { where } Y_{r, k}=1 \\
& L \leftarrow L-1 \\
& \text { else } \\
& E \leftarrow E \cup\{k\} \\
& k \leftarrow \arg \min _{j \in D} v_{c, j} \\
& \text { if }\|k\|>1 \text { then } \\
& k \leftarrow \text { resolve.tie.for.elimination }(k, X, v) \\
& \text { end if } \\
& F \leftarrow F \cup\{k\} \\
& \text { end if } \\
& D \leftarrow D \backslash\{k\} \quad \triangleright \text { Candidate } k \text { is removed from the pool of hopefuls } \\
& Y_{i, r} \leftarrow Y_{i, r}-1 \quad \forall i=1, \ldots, N \text { where } Y_{i, k}>0 \text { and } r=1, \ldots, M \text { where } Y_{i, r}>Y_{i, k} \\
& Y_{i, k} \leftarrow 0 \quad \forall i=1, \ldots, N \\
& \triangleright \text { Above: shift votes for voters who voted for candidate } k \\
& \text { end while } \\
& \operatorname{return}(E, F, v) \\
& \triangleright \text { Compute quota }
\end{aligned}
$$

Note: $\delta_{Y}(1)=1$ if $Y=1$ and 0 otherwise, is the Kronecker delta function; the arg max and arg min functions return sets, with more than one element when there is a tie; and $\|k\|$ is the number of elements in the set $k$.

Algorithm 1: STV algorithm. The input data consist of a matrix $X$ of the votes of size $N \times M$, with $N$ being the number of ballots and $M$ the number of candidates. $m$ is the number of seats to be filled and $\varepsilon$ is a small number used for defining the quota. 
total number of second preferences, then the third preferences, and so on. If a tie cannot be resolved even by counting the last preference, then it is broken by a random draw with equal probabilities for the tied candidates. A random seed is specified so that the result is replicable.

Combining Forwards and Backwards Tie-Breaking with the Ordered method and random sampling, each tie in the stv function is broken in one of the following three ways:

1. Forwards (" $\mathrm{f}$ ") or Backwards ("b") Tie-Breaking method alone

2. Forwards or Backwards Tie-Breaking followed by the Ordered method ("fo", "bo")

3. Forwards or Backwards Tie-Breaking followed by the Ordered method, and finally, random sampling ("fos", "bos")

The abbreviation of these three possibilities in parentheses is included in the STV output whenever a tie is broken during the election count.

Ties of any kind are relatively rare unless the electorate is small. In very small electorates, ties are more common, but cases where Forwards, Backwards, and Ordered Tie-Breaking all fail to break the tie are unusual even then, so election by random draw will be a rare event.

In the earliest version of the software (Silverman, 2002), ties were broken deterministically: if a candidate was to be elected, the last-named member of a tie was chosen. On the other hand, if there was a tie for elimination, it was the first-named who was eliminated. These choices were aimed at compensating in a small way for the tendency of candidates higher up the ballot paper to get more votes. However, they depended on position on the ballot paper, which might be viewed as somewhat arbitrary, and in the vote package, we have used a more systematic criterion.

\section{Equal preference STV}

Extant implementations of STV require that voters not give equal preferences (except among the candidates that they do not rank). However, Meek (1970) has pointed out that the single transferable vote system does not exclude this possibility and outlined how the votes might be counted. This has never been implemented before in software, to our knowledge, although it is used for the election of the Trustees of the John Muir Trust Wikipedia (2020c).

The basic idea is that if, for example, a voter gives their first preference to candidates $\mathrm{A}$ and $\mathrm{B}$, then the vote will be equally split between the two, giving half a vote to each. If A is elected, then the proportion of the half-vote for A corresponding to A's surplus will be transferred to their next highest preference. This will be B if B is still in contention, i.e., if B has not been elected or eliminated by that stage. Otherwise, it will be the remaining candidate with the next highest preference from that voter. Similarly, if A is eliminated, the half-vote for A will be fully transferred to their next highest remaining preference. This will be B if B is still in contention, or otherwise the candidate with the next highest preference.

The same principle applies if there are three or more equal preferences. For example, consider the case where there are three equal preferences $\mathrm{A}, \mathrm{B}$, and $\mathrm{C}$, and $\mathrm{A}$ is eliminated/elected. If $\mathrm{A}$ is elected, the proportion of the one-third vote for A corresponding to A's surplus is equally divided between B and C. If A is eliminated, then both B and C get increased to a half vote. Algebraically, this is implemented by the change below in Line 11 of Algorithm 1.

Otherwise, the count proceeds in the same way as when equal preferences are not allowed. The argument equal. ranking in the stv function is set to TRUE when equal preferences are allowed. In this case, votes are postprocessed before counting so that they correctly reflect preferences. For example, a vote $1,1,2,3,3,3$ would be recoded to $1,1,3,4,4,4$. This is in contrast with the usual case where equal preferences are not allowed and equal. ranking=FALSE when votes with non-sequential preferences, such as 1,2, 4, 5, are declared invalid and considered spoiled unless a partial correction is allowed.

STV with equal preferences can be implemented by Algorithm 1 with only two relatively small changes, namely:

Line 11 replaced by: $u_{i, j} \leftarrow w_{i} \delta_{Y_{i, j}}(1) / \sum_{\ell=1}^{M} \delta_{Y_{i, \ell}}(1) \quad \forall i=1, \ldots, N, j=1, \ldots, M$

Line 20 replaced by: $w_{r} \leftarrow \sum_{j=1}^{M} u_{r, j}-u_{r, k}+u_{r, k} * S \quad \forall r$ where $Y_{r, k}=1$

Note that if applied to votes with no equal preferences, the modified algorithm yields the same result as Algorithm 1. In such a case, the denominator in Line 11 is equal to 1 for all $i$, and thus, $u$ is the same as in Algorithm 1. Similarly, in Line 20, $\sum_{j=1}^{M} u_{r, j}-u_{r, k}=0$ if there are no equal preferences, and thus, $w$ is the same as in Algorithm 1.

We illustrate this functionality using the food election data by setting the first three votes to equal first preferences for Chocolate and Strawberries, instead of first and second preferences: 
$>$ food_election2 <- food_election

$>$ food_election2[c(1:3), 4]<- 1

$>\operatorname{stv}$ (food_election2, equal.ranking $=$ TRUE)

Results of Single transferable vote with equal preferences

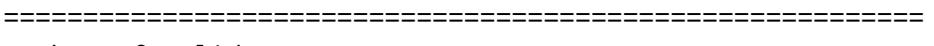

Number of valid votes: 20

Number of invalid votes: 0

Number of candidates: 5

Number of seats: 2

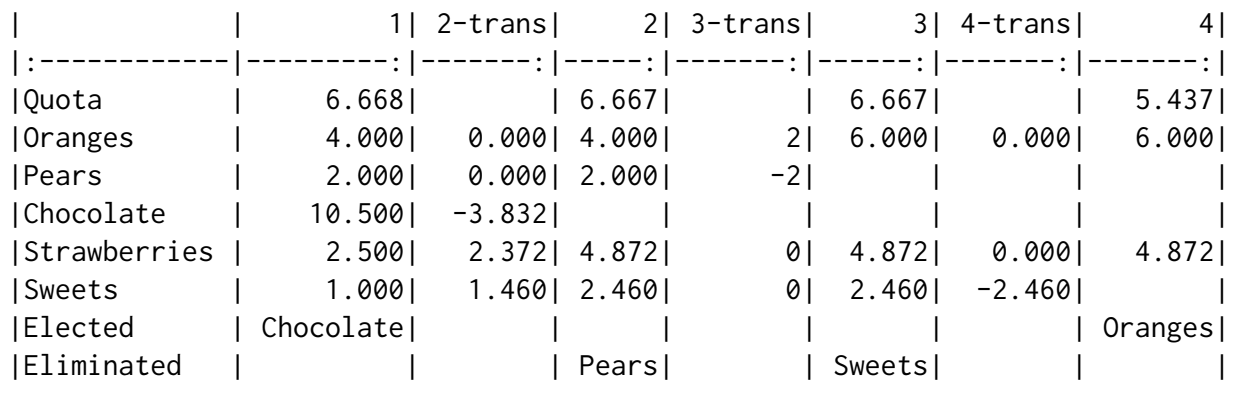

Elected: Chocolate, Oranges

Once again, Oranges is elected second, ahead of Strawberries, although the margin of victory is smaller than before.

\section{Reserved seats in STV}

In addition to having a given number of seats to fill, it may be desired to elect a minimum number of candidates from a specified class or group of candidates. For example, the selectors of plenary papers at a conference might wish to reserve at least two slots for students. Or the election of a committee might wish to ensure that at least three women were elected.

We have incorporated this feature into the stv function as an option. Users can specify the number of reserved seats with the argument group. nseats and mark the members eligible for those seats in the argument group. members.

When this requirement is present, we modify our STV algorithm as follows. Suppose $m$ denotes the number of seats and $g$ denotes the number of reserved seats, and candidates are either marked (eligible for reserved seats) or unmarked (not eligible). Then on each count,

- if the leading candidate exceeds the quota, they are elected, except that if $m-g$ unmarked candidates have already been elected, they are only elected if they are marked. Or,

- if no candidate has been elected on this round, the candidate with the fewest votes is eliminated, except that if there are only $g$ marked candidates still in play (including any already elected) or if there are already $m-g$ unmarked candidates elected, the unmarked candidate with the fewest votes is eliminated (even if that number of votes is above the quota).

We will illustrate the reserved seats feature in Section 2.4.3.

We are not aware of any previous method in the literature to allow for reserved seats in STV. It seems possible that other methods for doing this could be developed. For example, one could just eliminate all remaining unmarked candidates once $m-g$ unmarked candidates had been elected. It is not clear if or when this would give different results.

In principle, this general approach could be extended to the situation where there are two or more constraints, such as ensuring that at least three women and at least two students are elected to a committee of 12. The basic principles are the same:

- When eliminating a candidate, make sure that there are enough of the right kinds of candidates still in play that all remaining constraints can be satisfied.

- When choosing a candidate to elect, make sure there are enough slots left so that the remaining constraints can be satisfied later.

However, in the vote package, for now, it is implemented only for one constraint. 


\section{Examples}

We now illustrate the different systems using three examples of elections. Perhaps ironically, systems are more robust with larger than smaller electorates, in the sense that their results are less sensitive to small changes in the electoral system. We therefore start with a political election with a relatively large electorate, continue with the election of the council of a scientific organization with a moderatesized electorate, and finally describe an election with a very small electorate. Each of these was a multi-winner election, but we will also use them to illustrate the single-winner electoral methods.

\section{Irish general election 2002: Dublin West constituency}

The Dublin West constituency in the 2002 Irish general election had three seats to be filled, nine candidates, and just under 30,000 ranked votes. The dataset, called dublin_west, is included in the package. These data were collected electronically in a trial of electronic voting, with a system that prevented invalid votes. As a result, there were no invalid votes, unusually for an election of this kind.

$>$ data (dublin_west)

$>$ head(dublin_west)

Bonnie Burton Ryan Higgins Lenihan McDonald Morrissey Smyth Terry

$\begin{array}{llllllllll}1 & 0 & 4 & 0 & 3 & 0 & 0 & 1 & 5 & 2 \\ 2 & 0 & 0 & 2 & 0 & 1 & 4 & 3 & 0 & 0 \\ 3 & 0 & 0 & 3 & 0 & 1 & 0 & 2 & 0 & 0 \\ 4 & 0 & 2 & 0 & 0 & 0 & 0 & 3 & 0 & 1 \\ 5 & 0 & 2 & 1 & 0 & 0 & 0 & 0 & 0 & 0 \\ 6 & 0 & 3 & 2 & 0 & 1 & 0 & 0 & 0 & 0\end{array}$

We illustrate the single-winner methods by assuming that there is just one seat to be filled. First we consider the plurality method. It is necessary to convert the dataset into a set of zeros and ones to run the plurality function:

$>$ dublin_west1 <- $1 *($ dublin_west == 1$)$

$>$ plurality (dublin_west1)

Results of Plurality voting

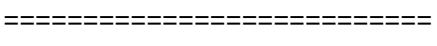

Number of valid votes: 29988

Number of invalid votes: 0

Number of candidates: 9

Number of seats: 1

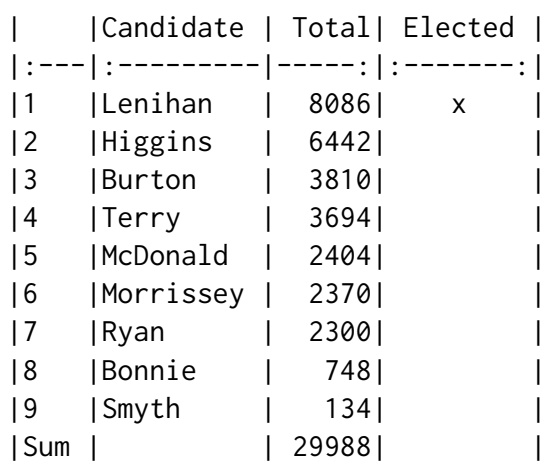

Elected: Lenihan

Lenihan was elected, although he received only $27 \%$ of the first preference votes.

Here is the two-round runoff result:

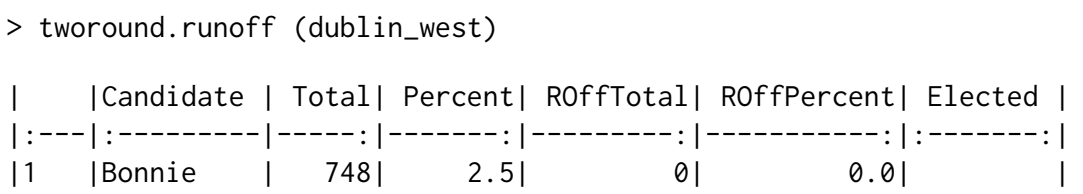




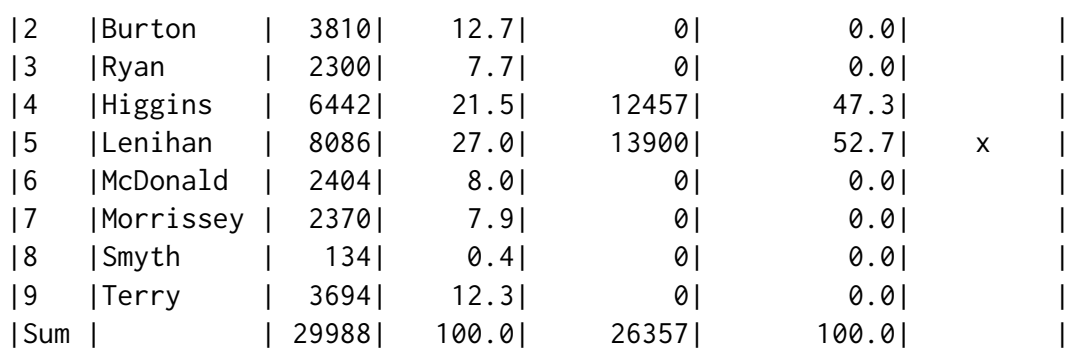

Elected: Lenihan

Lenihan was again elected, but this time after a run-off, as he did not get a majority on the first count. He got an absolute majority on the second count. This indicates a broader base of support than the plurality vote.

We now illustrate the single-winner approval voting method by assuming that voters "approved" any candidate to whom they gave their first, second, or third preference. Under this assumption, voters approved 2.8 candidates on average.

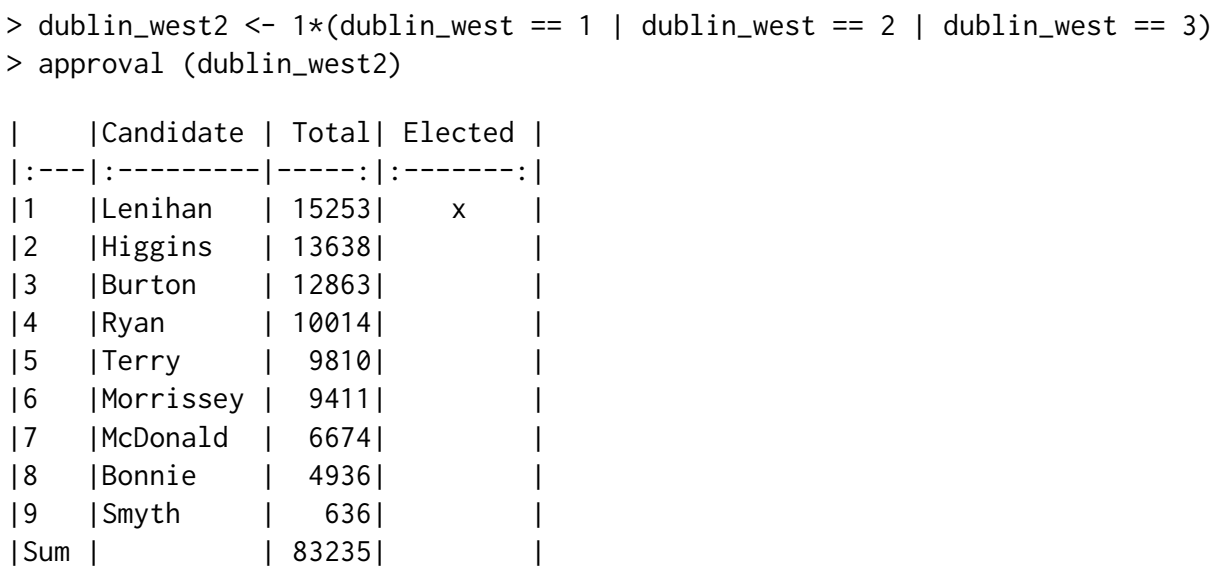

Elected: Lenihan

Once again, Lenihan wins. The multi-winner approval vote method with three seats gives wins to Lenihan, Higgins, and Burton because they got the most votes.

The Condorcet method did have both a winner and a loser in this case:

$>$ condorcet (dublin_west)

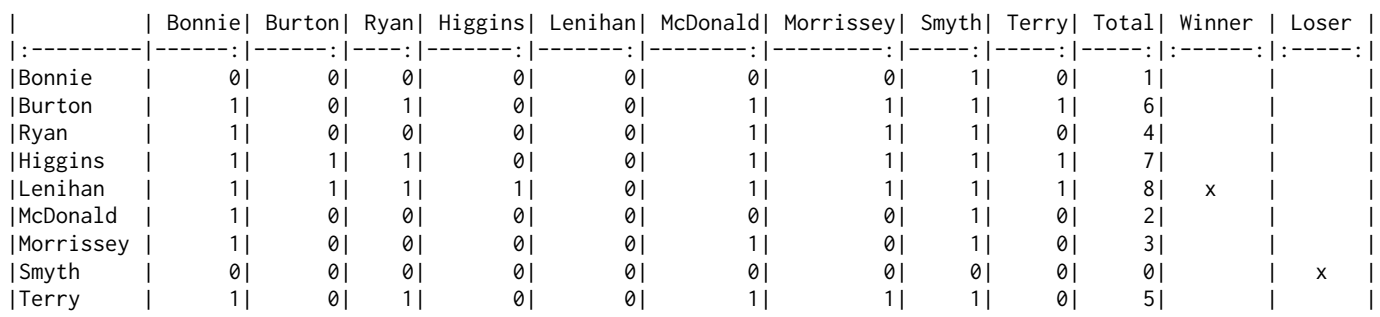

Condorcet winner: Lenihan

Condorcet loser: Smyth

The STV result is as follows:

$>$ stv.dwest $<-$ stv (dublin_west, nseats $=3$, eps $=1$, digits $=0$ )

Results of Single transferable vote

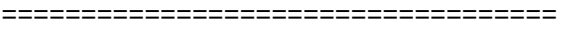

Number of valid votes: 29988

Number of invalid votes: 0

Number of candidates: 9

Number of seats: 3 


\begin{tabular}{|c|c|c|c|c|c|c|c|c|c|c|c|c|c|c|c|}
\hline & 11 & -trans | & 21 & 3-trans & 31 & trans $\mid$ & 41 & 5-trans| & 51 & 6-trans $\mid$ & 61 & 7-trans $\mid$ & 71 & 8 -trans 1 & 81 \\
\hline Quota & $7498 \mid$ & 1 & 74911 & 1 & 74861 & 1 & 7465 & 1 & 7303 & 1 & 7233 & & 7043 & 1 & 6143 \\
\hline Bonnie & 7481 & 81 & 7561 & 201 & $776 \mid$ & $-776 \mid$ & & i & । & । & 1 & i & i & 1 & \\
\hline |Burton & $3810 \mid$ & 55 & 3865 & $4 \mid$ & $3869 \mid$ & $207 \mid$ & $4076 \mid$ & 2951 & 4372 & 211| & $4583 \mid$ & $763 \mid$ & 5345 & $1191 \mid$ & $6536 \mid$ \\
\hline Ryan & 23001 & 2981 & $2598 \mid$ & $23 \mid$ & 26211 & 651 & $2686 \mid$ & 3571 & $3042 \mid$ & $77 \mid$ & $3119 \mid$ & $673 \mid$ & $3792 \mid$ & $-3792 \mid$ & \\
\hline |Higgins & $6442 \mid$ & 681 & 6510 & $21 \mid$ & 65311 & 1981 & $6728 \mid$ & $1124 \mid$ & $7853 \mid$ & $-550 \mid$ & i & i & i & i & \\
\hline Lenihan & $8086 \mid$ & $-588 \mid$ & & i & & i & & & i & i & i & i & i & i & \\
\hline McDonald & $2404 \mid$ & 241 & $2428 \mid$ & 19 & $2447 \mid$ & 761 & $2523 \mid$ & $-2523 \mid$ & । & i & & 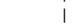 & 1 & i & \\
\hline Morrissey & $2370 \mid$ & 701 & 2440 & 131 & 2453 & 981 & $2551 \mid$ & 1081 & 2659 & 52 & 27111 & $-2711 \mid$ & i & i & \\
\hline Smyth & $134 \mid$ & 11 & 135 & $-135 \mid$ & & i & & । & । & i & । & & i & i & \\
\hline |Terry & 3694 & $43 \mid$ & $3737 \mid$ & $21 \mid$ & $3758 \mid$ & 691 & $3828 \mid$ & $151 \mid$ & 3979 & $71 \mid$ & 40501 & $896 \mid$ & $4946 \mid$ & 8021 & $5748 \mid$ \\
\hline Elect & | Lenihan | & 1 & & & & & & & iggins | & & & & & & Burton| \\
\hline Eliminated & & & Smyth| & & Bonnie| & & onald| & i & i & & isseyl & & Ryan | & & \\
\hline
\end{tabular}

Elected: Lenihan, Higgins, Burton

The three candidates elected were also the ones who got the most first preference votes. All the candidates represented different political parties or were independents, except Ryan and Lenihan, who were both candidates for the Fianna Fáil party, the largest party in Ireland at the time. Lenihan was elected on the first count with a surplus of 588 votes, and 298 of these were transferred to Ryan, the most of any candidate. This reflects the fact that voters tend to give their highest preferences to candidates of the same party, although here, we can see that many of the Lenihan voters did not, in fact, give their second preferences to Ryan.

Although this was an election with almost 30,000 votes and the electoral system appears somewhat complex, the counting takes just two seconds on a Macbook Pro laptop.

Note that the results were slightly different from the results using the Irish STV system, although the same candidates were elected; see Wikipedia (2020b). This is because of several minor differences between the Irish STV system and the stv function in the vote package. The most important of these is that in the vote package, the quota declines as the counts proceed to reflect votes that are not transferred because voters did not express enough preferences. In the Irish STV system, the quota remains the same throughout the counts. We chose to make the quota adaptive because it allows a more complete transfer of the votes of candidates elected. However, if the argument constant. quota is set to TRUE, the quota is kept constant for all counts.

The STV results can be visualized in several ways. Figure 1 has been produced by the command $>$ plot (stv.dwest)

It shows the evolution of the candidate's vote totals over successive counts, as well as of the quota. It can be seen that while candidates mostly stayed in the same order, candidate Ryan overtook two other candidates thanks to transfers, even though she was eventually eliminated. This reflects the fact that she had high preferences among voters who gave their first preferences to Lenihan and Morrissey.

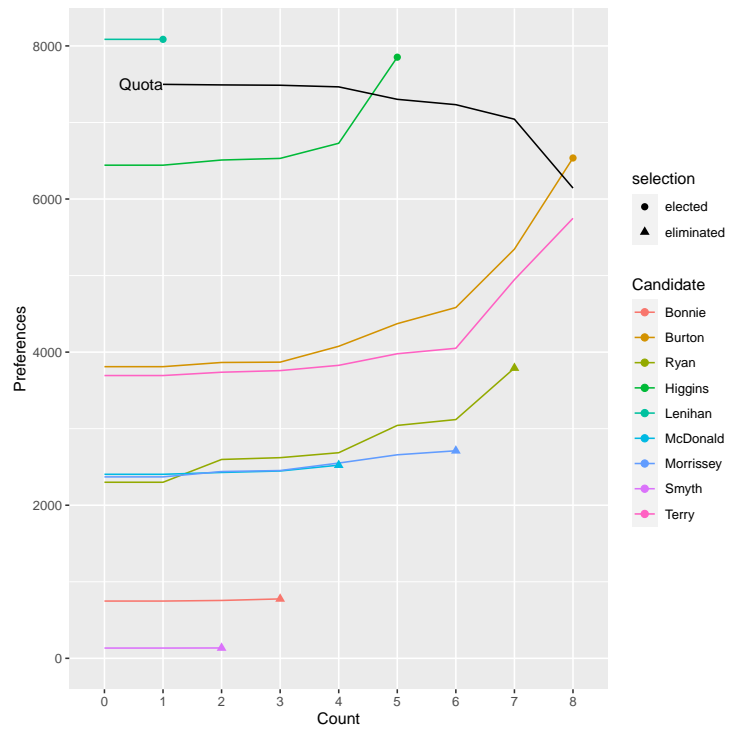

Figure 1: Evolution of candidates' votes over STV counts in the 2002 Irish general election in Dublin West.

Figure 2 shows the number of preference votes that each candidate received. The first preferences reflect the numbers we know from the first count. It can be seen that Ryan and Burton had the most second preferences; in Ryan's case, this is because she was the second Fianna Fáil (FF) candidate behind Lenihan and got the majority of his second preferences. Burton and Morrissey had the most third preferences. 


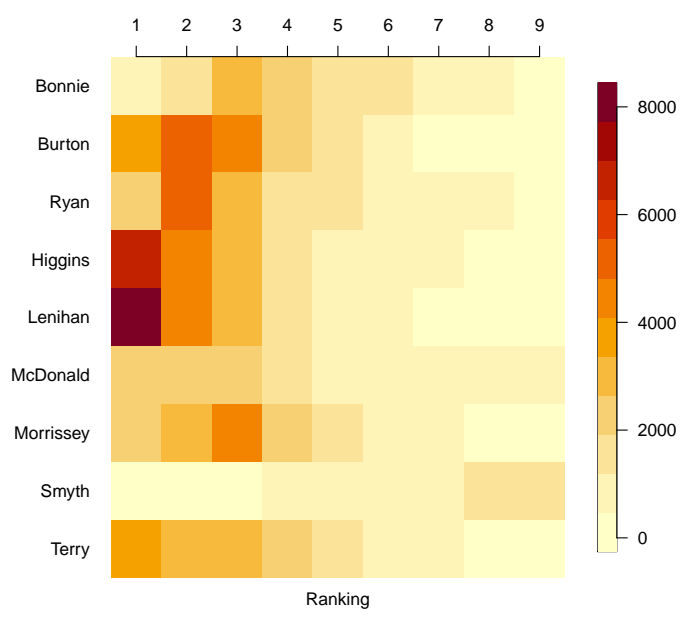

Figure 2: Number of preference votes that each candidate received in the 2002 Irish general election in Dublin West.

Figure 3 (left panel) shows the number of votes for each combination of first and second preference. The biggest number is those who voted first for Lenihan and then for Ryan, again reflecting that they are from the same party and that Lenihan had the most first preferences.
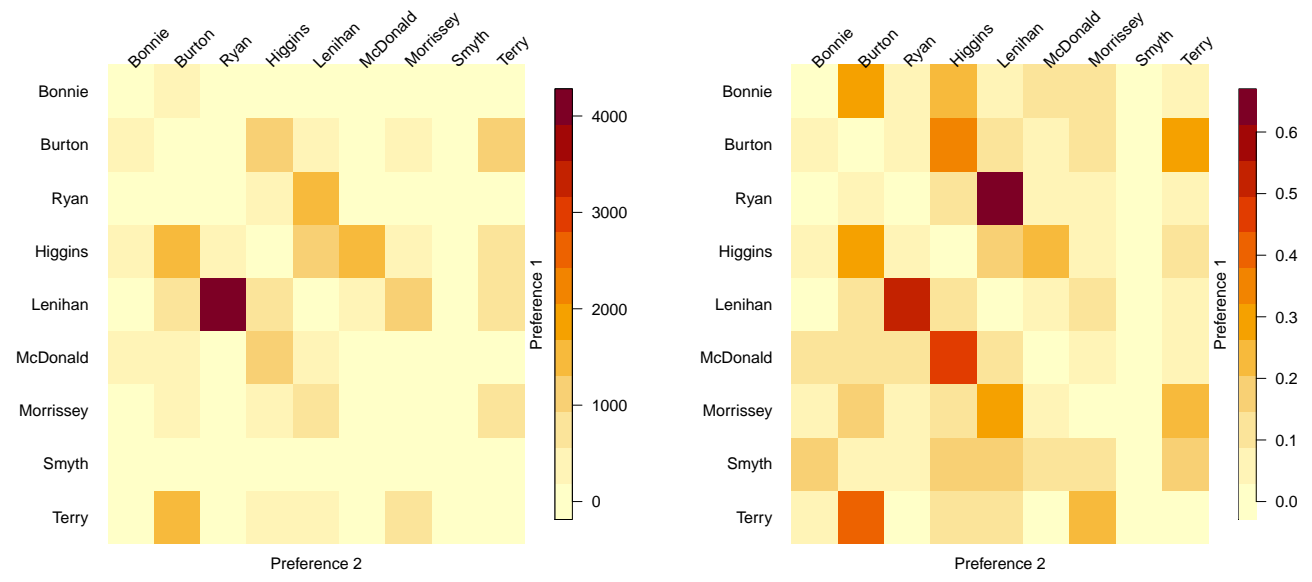

Figure 3: Joint preferences in the 2002 Irish general election in Dublin West. Left panel: Number of votes for each combination of first and second preferences. Right panel: Proportion of the first preference votes for each candidate that gave their second preference vote to each other candidate.

The right panel in Figure 3 shows the same information but in the form of the proportion of the first preference voters for each candidate that cast their second preference votes for each other candidate. The largest single cell shows that over $60 \%$ of Ryan voters cast their second preferences for Lenihan.

The code for producing Figures 2 and 3 is as follows:

$>$ image (stv.dwest, all.pref = TRUE) \# Figure 2
$>$ image (stv.dwest, proportion = FALSE) \# Figure 3 left panel
$>$ image (stv.dwest, proportion = TRUE) \# Figure 3 right panel

Note that the image method is available for all functions in the package that use ranked votes, namely condorcet and tworound.runoff, in addition to stv. However, the method cannot be used if equal preferences are present in the ballots. 


\section{IMS council election}

The ims_election dataset contains the votes in a past election for the Council of the Institute of Mathematical Statistics (IMS). There were four seats to be filled with 10 candidates running and 620 voters. The names of the candidates have been anonymized ${ }^{2}$. The election was carried out by STV. The results were:

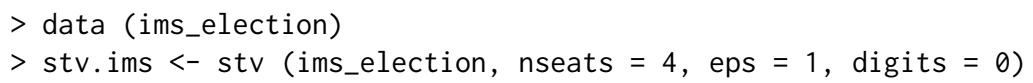

The results are shown in Figure 4. Although the electorate was much smaller, the results show some common patterns to those from Dublin West. The quota declined slowly in the early counts and more rapidly in the later ones. The four candidates elected were the ones that got the most first preferences. Figure 4(d) shows that, while there are no political parties in this election, Tilmann and Hillary tended to share voters, as did Jasper and Li. We do not know the identities of the candidates because their names have been anonymized, but these pairs of candidates clearly appeal to the same voters, perhaps because of geographical or intellectual commonalities.

However, neither Li nor Hillary was able to benefit from these shared preferences in this election. While Jasper was elected on the second count, he reached exactly the number of votes needed to reach the quota, namely 119, and thus no surplus was available for a transfer. Tilmann, on the other hand, was elected last, after which the election ended. If there had been one more seat available (i.e., nseats = 5), Hillary would have got Tilmann's surplus and then would have been elected.

In this example, 29 votes were identified as invalid. One can explore those votes using

$>$ invalid.votes(stv.ims)

Most of these votes are all zero preference votes. However, a few of them contain a gap in the ranking. If it is desired that such votes be considered valid up to the last valid ranking, one can add the argument invalid. partial = TRUE to the stv call. In this case, those votes are corrected. Using corrected.votes(stv.ims) will then display the original and corrected versions of the votes. Similarly, valid.votes (stv.ims) will display all the valid votes considered in the election.

\section{Trial faculty recruitment vote}

This is a trial election that was carried out to test a proposed use of STV in a university statistics department for selecting faculty job candidates to whom to make offers. There were two jobs to be filled, five finalists, and ten voters. It was desired to select the two candidates to whom to make offers and also to produce a ranking of the other candidates. This is fairly typical of such elections. The candidates were named Augustin-Louis Cauchy, Carl Friedrich Gauss, Pierre-Simon Laplace, Florence Nightingale, and Siméon Poisson.

The voters entered their choices into a web-based survey which was then converted into a text file. Here, we create the corresponding dataset manually:

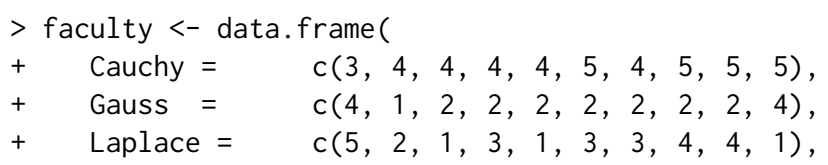

\footnotetext{
${ }^{2}$ To ensure confidentiality, the names of the candidates were replaced by arbitrarily chosen first names that have no connection to the actual names of the candidates.
} 

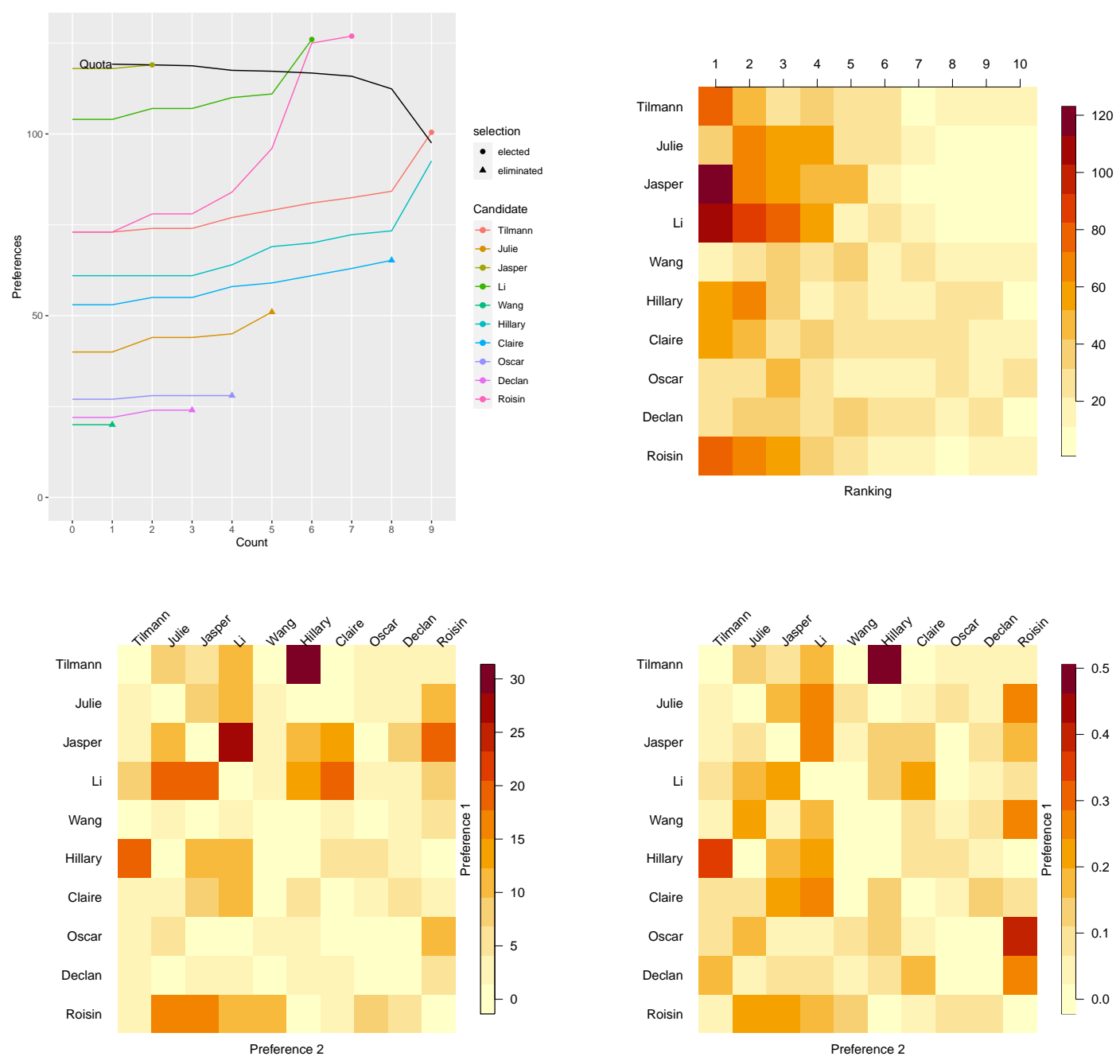

Figure 4: Visualization of results of IMS Council election by STV. (a) Top left: Evolution of votes over counts. (b) Top right: Number of votes for each candidate at each preference level. (c) Bottom left: Number of votes for each first and second preference combination. (d) Bottom right: Number of second preferences as a proportion of the number of first preference voters for each candidate.

$+\quad$ Nightingale $=c(1,3,5,1,3,1,5,1,1,2)$,

+ Poisson $=c(2,5,3,5,5,4,1,3,3,3)$

+ )

The results of the STV election were as follows:

$>$ stv.faculty <- stv (faculty, nseats $=2$, digits $=2$, complete. ranking $=$ TRUE)

Results of Single transferable vote

\begin{tabular}{|c|c|c|c|c|c|c|c|c|}
\hline \\
\hline \multicolumn{9}{|c|}{$\begin{array}{ll}\text { Number of valid votes: } & 10 \\
\text { Number of invalid votes: } & 0\end{array}$} \\
\hline \multicolumn{9}{|c|}{ Number of candidates: } \\
\hline \multicolumn{9}{|c|}{ Number of seats: } \\
\hline I & I & $1 \mid$ & -trans & 21 & $3-\operatorname{trans}$ & 31 & -trans & $4 \mid$ \\
\hline |:--- & $-1-$ & $-: 1$ & $---: \mid-$ & $----: \mid$ & $------: \mid-$ & $---: \mid-$ & $----: \mid-$ & $---: \mid$ \\
\hline IQuota & I & 3.331 & | & 3.331 & I & 3.331 & | & 3.331 \\
\hline |Cauchy & | & 0.001 & 0.001 & 0.001 & 이 & | & | & 1 \\
\hline |Gauss & | & $1.00 \mid$ & $1.33 \mid$ & 2.331 & 01 & 2.331 & $1.33 \mid$ & $3.67 \mid$ \\
\hline |Laplace & | & 3.001 & 0.001 & 3.001 & 01 & 3.001 & 0.001 & 3.001 \\
\hline
\end{tabular}




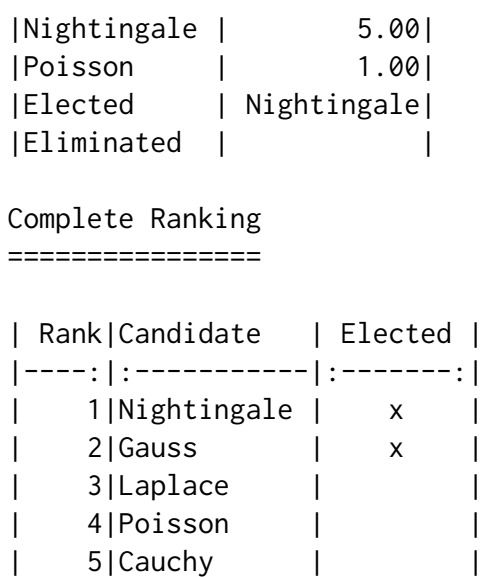

Elected: Nightingale, Gauss

Nightingale and Gauss were elected. The complete ranking could be useful for a vote like this, where an ordering beyond the winning candidates may be desired, for example, to make further offers if one of the top two declines the offer. Note that the complete ranking is conditional on the pre-specified number of seats or winners in the election.

The results are illustrated in Figure 5. An interesting feature that can be seen from Figure 5(a) is that Laplace got more first preference votes than Gauss, but Gauss ended up beating him by a small margin for the second offer because almost every voter gave Gauss either their first or second preference. Thus, as other candidates were elected or eliminated, their votes were transferred to Gauss rather than Laplace. The large number of second preferences for Gauss is apparent from Figure 5(b). Figure 5(c) and especially Figure 5(d) show that Gauss got the highest number and proportion of second preference votes from the electors of each of the other candidates.

If this had been done by approval voting, and all the voters had approved their top two choices, the same two candidates would have been selected as by STV (i.e., Nightingale and Gauss).

It is interesting to note that there was no Condorcet winner in this election, even though Nightingale was far ahead of the other candidates by most criteria:

\begin{tabular}{|c|c|c|c|c|c|c|c|}
\hline I & | Cauchy | & Gauss | & Laplace | & Nightingale| & Poisson| & Total| & Loser \\
\hline . & $-|-----:|-$ & $-----: \mid$ & $|------:|$ & $----------: \mid$ & $-------: \mid-$ & $-----: \mid$ & $:----$ \\
\hline ICauchy & 이 & 01 & 01 & 01 & 01 & 01 & $x$ \\
\hline |Gauss & $1 \mid$ & 01 & $1 \mid$ & 01 & $1 \mid$ & 31 & \\
\hline |Laplace & $1 \mid$ & 01 & 01 & 01 & $1 \mid$ & 21 & \\
\hline |Nightingale & $1 \mid$ & $1 \mid$ & 01 & 01 & $1 \mid$ & 31 & \\
\hline Poisson & $1 \mid$ & 01 & 01 & 01 & 01 & 11 & \\
\hline
\end{tabular}

There is no condorcet winner (no candidate won over all other candidates). Condorcet loser: Cauchy

This illustrates the fact that even in a relatively clearcut case, there may be no Condorcet winner.

To illustrate the feature of reserved seats in STV, let us assume that it is required that at least one French candidate be selected. Then,

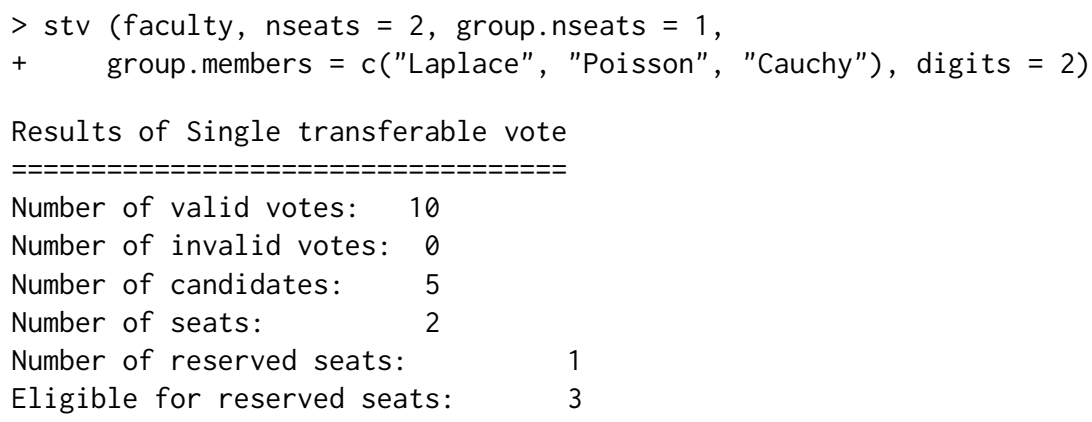




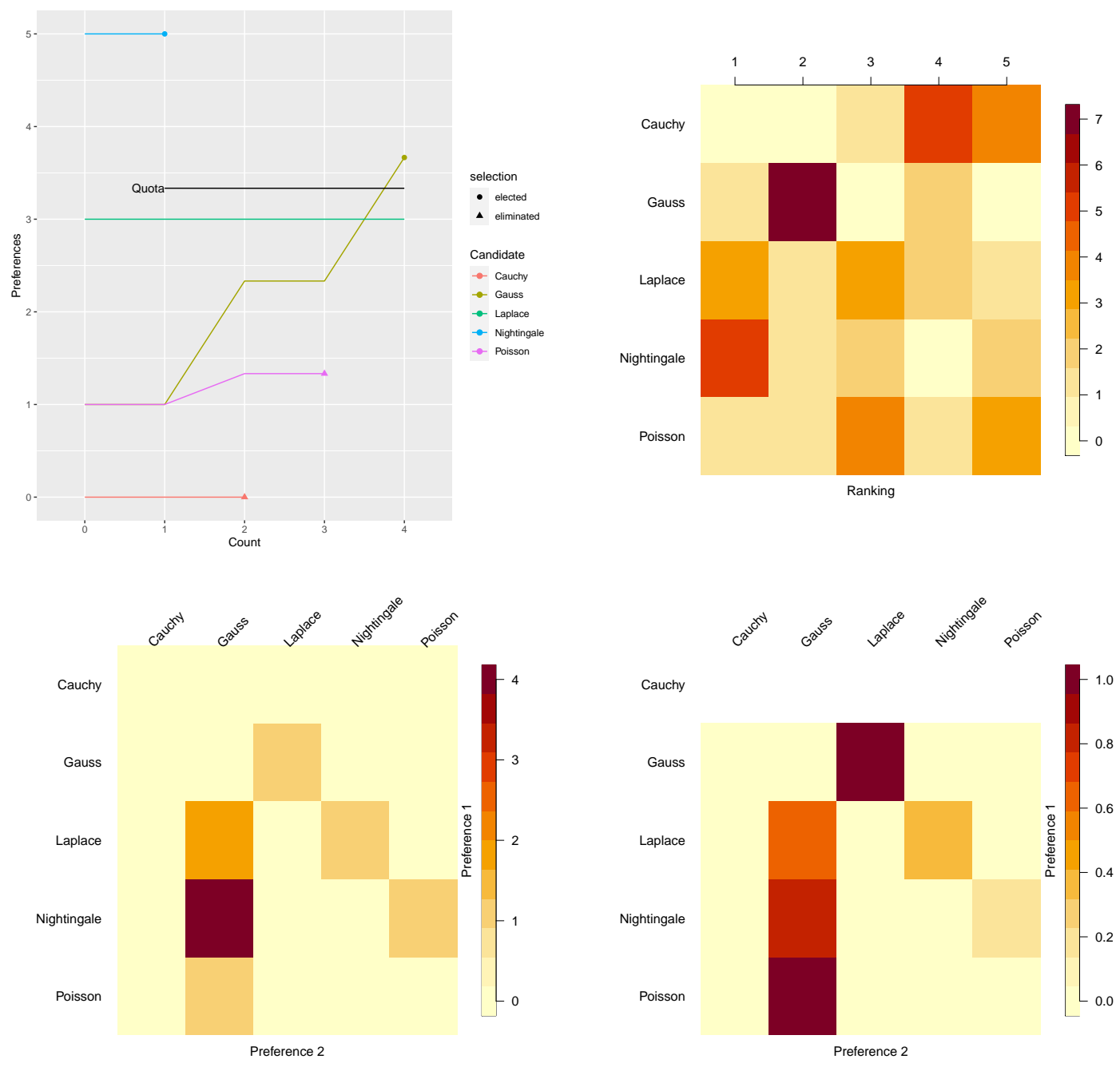

Figure 5: Visualization of results of the trial faculty recruitment vote by STV. (a) Top left: Evolution of votes over counts. (b) Top right: Number of votes for each candidate at each preference level. (c) Bottom left: Number of votes for each first and second preference combination. (d) Bottom right: Number of second preferences as a proportion of the number of first preference voters for each candidate.

\begin{tabular}{|c|c|c|c|c|c|}
\hline Quota & 3.331 & । & 3.331 & i & 3.331 \\
\hline |Cauchy* & 0.001 & 0.001 & 0.001 & 0.001 & 0.001 \\
\hline |Gauss & $1.00 \mid$ & $1.33 \mid$ & $2.33 \mid$ & $-2.33 \mid$ & \\
\hline |Laplace* & 3.001 & 0.001 & 3.001 & $1.67 \mid$ & 4.67 \\
\hline |Nightingale & 5.001 & $-1.67 \mid$ & 1 & | & \\
\hline |Poisson* & $1.00 \mid$ & 0.331 & $1.33 \mid$ & $0.67 \mid$ & 2.00 \\
\hline |Elected & | Nightingale| & | & | & & Laplace \\
\hline |Eliminated & 1 & & Gauss | & I & \\
\hline
\end{tabular}

Elected: Nightingale, Laplace

Here, the modifications to the algorithm described in Section 2.3.5 ensured that none of the French candidates was eliminated on the second count, as the only seat left at that point was the reserved seat. Thus, Gauss, the only non-French candidate left, was eliminated in spite of having more votes than Cauchy or Poisson. Laplace was then elected on the following count. In the output, the candidates eligible for reserved seats are marked with a star.

We now modify this dataset slightly to illustrate the equal ranking STV method. Four of the votes were changed so as to include equal preferences: 


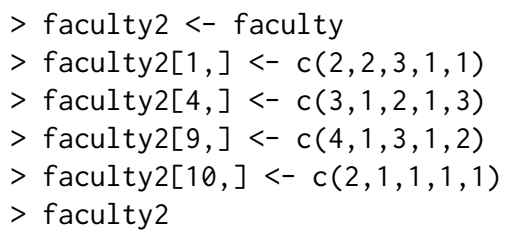

$\begin{array}{lrrrrr} & \text { Cauchy } & \text { Gauss } & \text { Laplace } & \text { Nightingale } & \text { Poisson } \\ 1 & 2 & 2 & 3 & 1 & 1 \\ 2 & 4 & 1 & 2 & 3 & 5 \\ 3 & 4 & 2 & 1 & 5 & 3 \\ 4 & 3 & 1 & 2 & 1 & 3 \\ 5 & 4 & 2 & 1 & 3 & 5 \\ 6 & 5 & 2 & 3 & 1 & 4 \\ 7 & 4 & 2 & 3 & 5 & 1 \\ 8 & 5 & 2 & 4 & 1 & 3 \\ 9 & 4 & 1 & 3 & 1 & 2 \\ 10 & 2 & 1 & 1 & 1 & 1\end{array}$

The results of the STV election with equal preferences were as follows:

$>$ stv.faculty. equal <- stv (faculty2, equal. ranking = TRUE, digits $=2$ )

Results of Single transferable vote with equal preferences

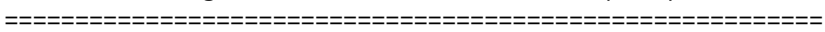
Number of valid votes: 10

Number of invalid votes: 0

Number of candidates: 5

Number of seats: 2

\begin{tabular}{|c|c|c|c|c|c|c|c|}
\hline | & \multicolumn{2}{|c|}{ 1| 2-trans| } & \multicolumn{2}{|c|}{$2|3-\operatorname{trans}|$} & \multicolumn{2}{|c|}{ 3| 4-trans| } & $4 \mid$ \\
\hline Ouota & 3.331 & 1 & 3.331 & 1 & 3331 & 1 & 3.33 \\
\hline | Cauchy & 0.001 & 0.001 & 0.001 & 01 & -1 & | & \\
\hline Gauss & $2.25 \mid$ & 0.341 & $2.59 \mid$ & 01 & 2.591 & 1.691 & $4.28 \mid$ \\
\hline |Laplace & $2.25 \mid$ & 0.011 & $2.26 \mid$ & 01 & $2.26 \mid$ & 0.131 & 2.391 \\
\hline Nightingale & $3.75 \mid$ & -0.421 & i & i & i & i & \\
\hline |Poisson & $1.75 \mid$ & 0.061 & $1.81 \mid$ & 01 & $1.81 \mid$ & $-1.81 \mid$ & \\
\hline |Elected & | Nightingale| & I & I & I & 1 & & Gauss | \\
\hline |Eliminated & I & & Sauchy| & & isson| & । & \\
\hline
\end{tabular}

Elected: Nightingale, Gauss

Warning message:

In correct.ranking (votes, quiet $=$ quiet) :

Votes 1, 4, 9, 10 were corrected to comply with the required format.

The warning message indicates that the ranking was corrected. When equal. ranking=TRUE, this correction will be made with any input, as long as the preferences are recorded as positive numbers (not necessarily integers). The corrected votes can be viewed using the corrected.votes function, which returns a list. The element new contains the updated votes:

$>$ corrected.votes(stv. faculty. equal)\$new

$\begin{array}{lrrrrr} & \text { Cauchy } & \text { Gauss } & \text { Laplace } & \text { Nightingale } & \text { Poisson } \\ 1 & 3 & 3 & 5 & 1 & 1 \\ 4 & 4 & 1 & 3 & 1 & 4 \\ 9 & 5 & 1 & 4 & 1 & 3 \\ 10 & 5 & 1 & 1 & 1 & 1\end{array}$

Such a correction is not made in the default case in which equal. ranking=FALSE, when the preferences have to be an ordered sequence of integers starting at one, with no ties and no gaps. However, votes can be corrected in the same way also from outside stv, using the function correct. ranking. As noted previously, another option is to set invalid. partial=TRUE in the stv function, which accepts partial-valid ranking, i.e., each vote is considered valid up to its largest valid preference, after which ties and gaps are set to 0 . When doing such correction externally via the correct. ranking function, set the argument partial to TRUE.

Finally, we give the results when there is a single winner to illustrate tie-breaking, as it so happens that tie-breaking is needed on two different counts in this case: 


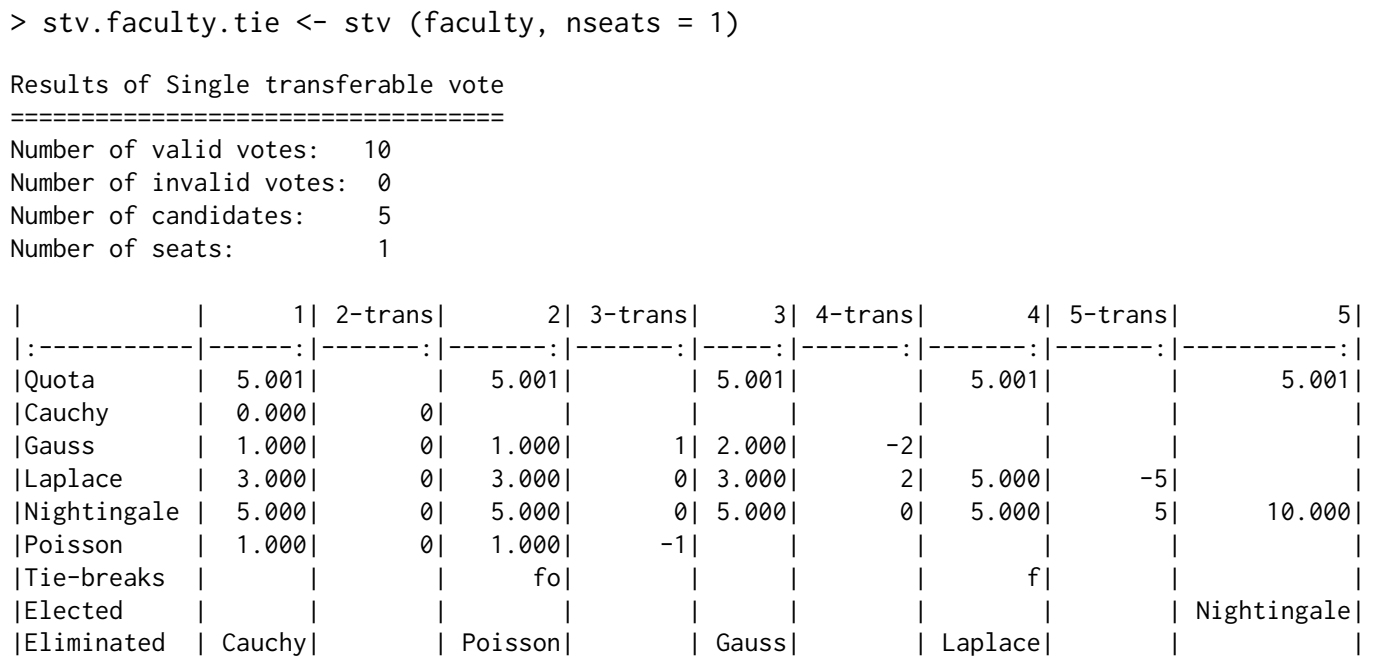

Elected: Nightingale

On the second count, Gauss and Poisson both had one vote, the lowest number, and so were tied for elimination. The Forwards Tie-Breaking method did not break the tie, as they both had the same number of votes also on the first count. The Ordered method did break the tie, however, because Gauss had 7 second preferences, and Poisson had only 1, so Poisson was eliminated. The notation "fo" in the Tie-breaks row indicates the tie-breaking method used, here Forwards followed be Ordered.

On the fourth count, Laplace and Nightingale were tied with 5 votes each, so they were tied for elimination as neither reached the quota of 5.001. The Forwards Tie-Breaking method was then used and involved looking first at their numbers of votes on the first count when Laplace had 3 votes, and Nightingale had 5. As a result, Laplace was eliminated, and then Nightingale was elected. If the Backwards Tie-Breaking method had been used (by setting ties = "b"), the comparison would have been done based on the third count instead of the first count. Here too, Laplace had 3 votes, and Nightingale had 5 on the third count, and thus, Laplace would have been eliminated.

Note that the ranking used by the Ordered method can be viewed via the ordered.tiebreak function, while passing the valid votes stored in the data element of the stv object:

$>$ ordered.tiebreak(stv.faculty.tie\$data)

$\begin{array}{crrrr}\text { Cauchy } & \text { Gauss } & \text { Laplace Nightingale } & \text { Poisson } \\ 1 & 3 & 4 & 5 & 2\end{array}$

attr (, "sampled")

[1] FALSE FALSE FALSE FALSE FALSE

It gives the elimination ranking. When used for electing a candidate, the order is reversed. The attribute "sampled" indicates for each candidate whether sampling was involved in determining its rank, which was not the case in our example. The function ordered.preferences can be used to view the matrix of preference counts from which the ordered ranking is derived. It gives the same information as the image plot with all. pref = TRUE, but in matrix form.

\section{Discussion}

We have described and illustrated the vote package in $\mathrm{R}$, which implements several electoral systems, namely the plurality, two-round runoff, approval, score, and Single Transferable Vote (STV) systems (Ševčíková et al., 2021). It also identifies the Condorcet winner and loser, if they exist. It implements the Single Transferable Vote system with equal preferences, the first time this has been implemented in software to our knowledge. It also provides several ways of visualizing the STV results.

We are not advocating any electoral system, and indeed it is well known that no one system satisfies all of a set of criteria that one might reasonably want to hold. Thus, which system one uses can depend on the purpose of the election. However, we are particularly interested in multi-winner elections with small electorates, such as committee and council elections in organizations, and the selection of multiple job candidates, award winners, or other choices by small "selectorates." Such elections are common, and there is no universally accepted method for conducting them. We have found the STV system to work well in practice for such elections, and so we have emphasized it here, giving several examples. 
For completeness, we note that the most widely used political voting system around the world is a party-list approach, where voters vote for a party rather than for individuals, and some mechanism is then used to fill the party slots allocated (Electoral Reform Society, 2020). Such systems are not relevant for the purposes of our primary interest.

There are several other $\mathrm{R}$ packages that implement electoral systems. The votesys package implements several electoral methods, including several that are not included in the vote package (Wu, 2018). It implements the Instant Runoff Voting (IRV), which is the special case of STV for single-winner elections, but it does not implement the full version of STV for multi-winner elections. The rcv package also implements IRV (calling it Ranked Choice Voting) but has been removed from CRAN (Lee and Yancheff, 2019).

The STV package implements the STV method (Emerson et al., 2019). The results are generally very similar to those from the stv function in the vote package. However, there are some minor differences that can lead to different results, particularly in elections with small electorates. Notably, in the STV package, all quotas, vote counts, and transfers are rounded to integers, which can lead to different results when the electorate is small. Also, in the STV package all tie-breaking is done at random, in contrast with the vote package, which uses forwards and backwards tie-breaking. Unlike the vote package, none of these other packages implements the STV method with equal ranking or allows for reserved positions for marked groups.

The HighestMedianRules implements voting rules electing the candidate with the highest median grade (Fabre, 2020b,a). The electoral and esaps packages compute various measures of electoral systems; in spite of their names, they do not implement electoral systems or voting rules (Albuja, 2020; Schmidt, 2018).

Acknowledgements: The research of Raftery and Ševčíková was supported by NIH grant R01 HD070936. The authors are grateful to Salvatore Barbaro and Brendan Murphy for helpful discussions and to an anonymous reviewer for helpful comments that improved the article.

\section{Bibliography}

Ace Project. The systems and their consequences, 2020. URL https://aceproject.org/ace-en/ topics/es/esd/default. [p674]

J. Albuja. electoral: Allocating Seats Methods and Party System Scores, 2020. URL https://CRAN.Rproject.org/package=electoral. R package version 0.1.2. [p694]

K. Arrow. Social Choice and Individual Values. Yale University Press, New Haven, Conn., 2nd edition, 1963. [p674]

S. J. Brams and P. C. Fishburn. Approval voting. American Political Science Review, 72:831-847, 1978. [p676]

S. J. Brams and P. C. Fishburn. Approval Voting. Birkhauser, Boston, Mass., 1983. [p677]

S. J. Brams and P. C. Fishburn. Approval Voting. Springer Science \& Business Media, 2nd edition, 2007. [p676]

M. de Condorcet. Essai sur l'Application de l'Analyse à la Probabilité des Decisions Rendues à la Pluralité des Voix. Imprimerie Royale, Paris, France, 1785. [p677]

Electoral Reform Society. Party list proportional representation, 2020. URL https: //www. electoralreform.org.uk/voting-systems/types-of-voting-system/party-list-pr. [p694]

J. Emerson, S. Chandra, and L. Orr. STV: Single Transferable Vote Counting, 2019. URL https: //CRAN. Rproject. org/package=STV. R package version 1.0.1. [p694]

A. Fabre. Tie-breaking the highest median: alternatives to the majority judgment. Social Choice and Welfare, 2020a. doi: https://doi.org/10.1007/s00355-020-01269-9. [p694]

A. Fabre. HighestMedianRules: Implementation of Voting Rules Electing the Candidate with Highest Median Grade, 2020b. URL https: //CRAN. R-project.org/package=HighestMedianRules. R package version 1.0. [p694]

Fair Vote. Multi-winner ranked choice voting, 2020. URL https://www. fairvote.org/multi_winner_ rcv_example. [p678] 
A. Gibbard. Manipulation of voting schemes: A general result. Econometrika, 41:587-601, 1973. [p674]

I. D. Hill. Some aspects of elections - To fill one seat or many (with discussion). Journal of the Royal Statistics Society, Series A (Statistics in Society), 151:243-275, 1988. [p677, 678]

I. D. Hill, B. A. Wichmann, and D. R. Woodall. Single transferable vote by Meek's method. Computer Journal, 30:277-281, 1987. [p673, 679]

E. Kitchener. A new way to break STV ties in a special case. Voting Matters, 20:9-11, 2005. URL http://www. votingmatters.org.uk/ISSUE20/I20P3.PDF. [p680]

J. Lee and M. Yancheff. rcv: Ranked Choice Voting, 2019. URL https://cran.r-project.org/src/ contrib/Archive/rcv/. R package version 0.2.1. [p694]

London Elects. Mayor of London \& London Assembly elections: Counting the votes, 2020. URL https://www. londonelects.org.uk/im-voter/counting-votes. [p676]

J. Lundell. Random tie-breaking in STV. Voting Matters, 22:1-6, 2006. URL http: //www. votingmatters . org. uk/ISSUE22/I22P1.pdf. [p680]

B. L. Meek. Une nouvelle approche du scrutin transférable. Mathématiques et Sciences Humaines, 25: 13-23, 1969. [p679]

B. L. Meek. Une nouvelle approche du scrutin transférable (fin). Mathématiques et Sciences Humaines, 29:33-39, 1970. [p679, 682]

R. A. Newland, F. S. Britton, C. Rosenstiel, and J. Woodward-Nutt. How to Conduct an Election by the Single Transferable Vote. Electoral Reform Society, London, U.K., 1997. URL https://www.electoral-reform.org.uk/latest-news-and-research/publications/ how-to-conduct-an-election-by-the-single-transferable-vote-3rd-edition. [p678, 680]

J. C. O'Neill. Tie-breaking with the single transferable vote. Voting Matters, 18:14-17, 2004. URL http://www. votingmatters.org.uk/ISSUE18/I18P6.PDF. [p680]

M. Satterthwaite. Strategy-proofness and Arrow's conditions: Existence and correspondence theorems for voting procedures and social welfare functions. Journal of Economic Theory, 10:187-217, 1975. [p674]

N. Schmidt. esaps: Indicators of Electoral Systems and Party Systems, 2018. URL https://CRAN.Rproject. org/package=esaps. R package version 0.1.0. [p694]

H. Ševčíková, A. E. Raftery, and B. W. Silverman. vote: Election Vote Counting, 2021. URL https: //CRAN. R-project.org/package=vote. R package version 2.3-0. [p673, 693]

B. W. Silverman. Single transferable voting system: Counting IMS elections by single transferable vote, 2002. URL https://imstat.org/elections/single-transferable-voting-system. [p673, $679,682]$

B. W. Silverman. Counting IMS elections: new procedure. IMS Bulletin, 32:3, 2003. [p673, 679]

E. Syddique. Discussion of 'Some aspects of elections - To fill one seat or many'. Journal of the Royal Statistical Society, Series A (Statistics in Society), 151:265, 1988. [p674]

N. Tideman. The single transferable vote. Journal of Economic Perspectives, 9:27-38, 1995. [p678]

Wikipedia. Condorcet method, 2020a. URL https://en.wikipedia.org/wiki/Condorcet_method. [p678]

Wikipedia. Dublin West Dáil constituency, 2020b. URL https://en.wikipedia.org/wiki/Dublin_ West\#2002_general_election. [p686]

Wikipedia. Single transferable vote, 2020c. URL https://en.wikipedia.org/wiki/Single transferable_vote. [p674, 682]

J. Wu. votesys: Voting Systems, Instant-Runoff Voting, Borda Method, Various Condorcet Methods, 2018. URL https: //CRAN. R-project. org/package=votesys. R package version 0.1.1. [p694] 
Adrian E. Raftery

Departments of Statistics and Sociology

University of Washington

Box 354322

Seattle, WA 98195-4322, USA

ORCID: 0000-0002-6589-301X

raftery@uw.edu

Hana Ševčíková

Center for Statistics and the Social Sciences

University of Washington

Box 354320

Seattle, WA 98195-4329, USA

ORCID: 0000-0002-7896-1704

hanas@uw.edu

Bernard W. Silverman

Rights Lab

University of Nottingham

Highfield House

University Park

Nottingham, NG7 2RD, U.K.

ORCID: 0000-0002-4059-2376

bernard.silverman@stats.ox.ac.uk 